\title{
SUBDIRECT SUM DECOMPOSITIONS OF ENDOMORPHISM RINGS
}

\section{John J. Hutchinson AND Julius Zelmanowitz}

The main result of this paper is that if a ring $R$ is an essential subdirect sum of prime rings and $M$ is a torsionless $R$-module, then the endomorphism ring of $M$ is also an essential subdirect sum of prime rings.

In [4] Levy defined irredundant subdirect sums of rings. This provided a unique decomposition of a left quotient semisimple ring as a subdirect sum of left quotient simple rings. He also showed that irredundant subdirect sums of prime rings possess some rather strong uniqueness properties.

In [2] essential subdirect sums were defined and Levy's decomposition theorem was obtained for essential subdirect sums. Furthermore in [3] it was shown that a ring $R$ has a maximal left quotient ring which is a direct product of full linear rings if and only if $R$ is an essential subdirect sum of rings whose maximal left quotient ring is full linear.

In $\S 1$ of this paper we show that for subdirect sums of prime rings, essential and irredundant subdirect sums are identical. We also give some uniqueness properties of essential subdirect sums which are even stronger than those for irredundant subdirect sums.

In $\S 2$ we show that if $M$ is a torsionless $R$-module and $R$ is an essential subdirect sum of prime rings, then the endomorphism ring of $M$ is also an essential subdirect sum of prime rings. We get this theorem as a consequence of a more general result about Morita contexts. The proof of the more general result is notationally more efficient.

1. Essential subdirect sums of prime rings. In what follows $R$ will be an associative ring which is not assumed to have an identity. ${ }_{R} M$ will express that $M$ is a left $R$-module. A submodule $K$ of $M$ is an essential submodule if every nonzero submodule of $M$ has nonzero intersection with $K . \quad M$ will then be an essential extension of $K$.

Let a set of nonzero rings $\left\{R_{\alpha} \mid \alpha \in A\right\}$ be given, and let $\left\{\pi_{\alpha} \mid \alpha \in A\right\}$ denote the set of projections from the (complete) direct product $\prod_{\alpha} R_{\alpha}$ onto $R_{\alpha} \cdot \quad R$ is a subdirect sum of the $R_{\alpha}$ if there is an isomorphism $f$ of $R$ into $\Pi_{\alpha} R_{\alpha}$ such that $f \pi_{\alpha}$ is onto $R_{\alpha}$ for each $\alpha \in A$.

Let $f_{\beta}$ be the map from $R$ into $\prod_{\alpha \neq \beta} R_{\alpha}$ defined by $f_{\beta}: r \rightarrow$ $\left\{r f \pi_{\alpha} \mid \alpha \neq \beta\right\}$. The subdirect sum is irredundant if the kernel of $f_{\beta}$ 
is nonzero for each $\beta \in A$. If we identify $R$ and $R f$ (which we will henceforth do), then $R \cong \Pi_{\alpha} R_{\alpha}$ and the subdirect sum is irredundant if and only if $R \cap R_{\alpha} \neq 0$ for all $\alpha \in A\left(R \cap R_{\alpha}=\operatorname{ker} f_{\alpha}\right)$.

The subdirect sum is essential if $R$ is an essential left $R$-submodule of $\Pi_{\alpha} R_{\alpha}$. If the subdirect sum is essential, then it is irredundant since $R_{\alpha}$ is an $R$-submodule of $\Pi_{\alpha} R_{\alpha}$ and $R_{\alpha} \cap R \neq 0$ for each $\alpha \in A$.

LEMMa 1.1. If $R$ is a subdirect sum of nonzero prime rings $\left\{R_{\alpha} \mid \alpha \in A\right\}$; then the subdirect sum is essential if and only if it is irredundant.

Proof. If the subdirect sum is irredundant, then by [4, Prop. 4.2] the maximal left quotient $\operatorname{ring} \bar{R}$ of $R$ is equal to the direct product of the maximal left quotient rings $\bar{R}_{\alpha}$ of $R_{\alpha}$. Since $R \cong$ $\Pi_{\alpha} R_{\alpha} \subseteq \Pi_{\alpha} \vec{R}$ it follows that ${ }_{R} R$ is essential in $\Pi_{\alpha} R_{\alpha}$ and the subdirect sum is essential.

The following example shows that essential subdirect sums and irredundant subdirect sums are not in general the same, even for left quotient semisimple rings.

ExAmple 1.2. Let $S_{1}$ and $S_{2}$ each be the direct sum of two copies of the integers. If

$$
R=\left\{((a, b),(c, d)) \in S_{1} \oplus S_{2} \mid b=d\right\},
$$

then $R$ is a ring which is an irredundant subdirect sum of $S_{1}$ and $S_{2}$. However $M=R x$ where $x=((0,0),(0,1))$ is a nonzero $R$-submodule of $S_{1} \oplus S_{2}$ but $M \cap R=0$. Hence $R$ is not an essential subdirect sum of $S_{1}$ and $S_{2}$.

Lemma 1.3. Suppose $R$ is a subdirect sum of nonzero rings $R_{1}, \cdots, R_{n}$ and each $R_{i}$ is a subdirect sum of $Q_{i, 1}, \cdots, Q_{i, k_{i}}$. The natural subdirect sum decomposition of $R$ as a subdirect sum of the $\left\{Q_{i, j}\right\}$ is essential if and only if $R$ is an essential subdirect sum of $R_{1}, \cdots, R_{n}$ and each $R_{i}$ is an essential subdirect sum of $Q_{i, 1}, \cdots, Q_{i, k_{i}}$.

Proof. [2, Prop. 2.2].

Loosely speaking, we may think of the preceding lemma as a generalized associative law for essential subdirect sums. The following example shows that irredundant subdirect sums do not have this property.

Example 1.4. Suppose $S_{1}, S_{2}$, and $R$ are as in Example 1.2, and 
let $S_{3}=S_{1}$. If $Q=\left\{(((a, b),(c, d)),(e, f)) \mid((a, b),(c, d)) \in R,(e, f) \in S_{3}\right.$, and $a=e\}$, then $Q$ is a ring and $Q$ is an irredundant subdirect sum of $R$ and $S_{3}$. The natural subdirect sum of $S_{1}, S_{2}$, and $S_{3}$ is not irredundant since $S_{1} \cap Q=0$.

Lemma 1.5. Let $C$ be an essential subdirect sum of prime rings $C_{1}, \cdots, C_{n}$ and let $A$ and $B$ be prime rings. If an essential subdirect sum of $A$ and $C$ is isomorphic to an essential subdirect sum of $B$ and $C$, then $A \cong B$.

Proof. If an essential subdirect sum of $A$ and $C$ is isomorphic to an essential subdirect sum of $B$ and $C$, then since $C$ is an essential subdirect sum of $C_{1}, \cdots, C_{n}$ we have by Lemma 1.3 that an essential subdirect sum of $A, C_{1}, \cdots, C_{n}$ is isomorphic to an essential subdirect sum of $B, C_{1}, \cdots, C_{n}$. By Lemma 1.1 the subdirect sum is irredundant, and by [4, Theo. 3.2] the subdirect summands are unique up to isomorphism. Thus $A \cong B$.

Loosely speaking, the preceding lemma is a cancellation law for essential subdirect sums of prime rings. The following example [4, p. 74] shows that the corresponding result does not hold for irredundant subdirect sums.

EXAMPLE 1.6. Let $A$ be a polynomial ring in an infinite number of indeterminants over the integers, and let $B$ be the same polynomial ring over the rationals. Then there is a homomorphism $f$ (respectively $g$ ) of $A$ (respectively $B$ ) onto itself with a nonzero kernel. Let $C=A \oplus B$. Then $R=\{(x,(y, z)) \mid x \in A,(y, z) \in C, f(x)=y\}$ is a subdirect sum of $A$ and $C$. The subdirect sum is irredundant since if $x$ is any nonzero element of $\operatorname{ker} f$, then $(x,(0,0)) \in A \cap R$; and if $x$ is any nonzero element of $B$, then $(0,(0, x)) \in C \cap R$. However $R \cong C$ since the middle component can be omitted. Similarly we construct an irredundant subdirect sum of $B$ and $C$ which is isomorphic to $C$. Since $A$ is not isomorphic to $B$, we have the desired example.

2. Essential subdirect sum decompositions of endomorphism rings. Assume that $(R, M, N, E)$ is a Morita context [1]. That is, suppose ${ }_{R} M_{R}$ and ${ }_{E} N_{R}$ are bimodules with $R-R$ bimodule homomorphism

$$
(,): M \otimes{ }_{E} N \rightarrow R
$$

and $E-E$ bimodule homomorphism

$$
\text { [ , ]: } N \oplus_{R} M \rightarrow E
$$

satisfying 


$$
m_{1}\left[n_{1}, m_{2}\right]=\left(m_{1}, n_{1}\right) m_{2} \quad \text { and } \quad n_{1}\left(m_{1}, n_{2}\right)=\left[n_{1}, m_{1}\right] n_{2}
$$

for all $n_{i} \in N$ and $m_{i} \in M$.

ExAMPLE 2.1. Let ${ }_{n} M$ be any $R$-module with $E=\operatorname{Hom}_{R}(M, M)$ and $N=\operatorname{Hom}_{R}(M, R)$. Set $(m, f)=m f$ for $m \in M$ and $f \in N$ and define $[f, m]$ via $m_{1}[f, m]=\left(m_{1}, f\right) m$ for $m_{1} \in M$. Then $(R, M, N, E)$ is a Morita context which we will call the standard context for ${ }_{R} M$. It is easy to see that $M_{E}$ is faithful and $(M, f)=0$ implies $f=0$. It is also immediate that ${ }_{R} M$ is torsionless if and only if $(m, N)=0$ implies $m=0$.

Henceforth we assume that $(R, M, N, E)$ is a Morita context with $E \neq 0$. If $I$ is an ideal of $R$ define $I^{\prime}=\{e \in E \mid(M e, N) \subseteq I\} ; I^{\prime}$ is an ideal of $E$. Similarly for $J$ an ideal of $E$, $J^{\prime}=\{r \in R \mid[N r, M] \subseteq J\}$ is an ideal of $R$.

LEMma 2.2. Let $P$ be a prime ideal of $R$ which does not contain $(M, N)$. Then

(i) $P^{\prime}$ is a prime ideal of $E$, and

(ii) $P=P^{\prime \prime}$.

Proof. (i) If $X Y \subseteq P^{\prime}$ where $X$ and $Y$ are ideals of $E$, then $(M X Y, N) \subseteq P$ so that $(M X, N)(M X, N)=(M X[N, M] Y, N) \subseteq$ $(M X Y, N) \subseteq P$. Since $P$ is prime, either $(M Y, N) \subseteq P$ or $(M Y, N) \subseteq$ $P$. Hence either $X \subseteq P^{\prime}$ or $Y \subseteq P^{\prime}$ and $P^{\prime}$ will be a prime ideal of $E$ provided $P^{\prime} \neq E$. Since $(M, N) \nsubseteq P,(M[N, M], N)=(M, N)^{2} \nsubseteq P$, and this implies that $P^{\prime} \neq E$.

(ii) It is easy to show that $P^{\prime \prime}=\{r \in R \mid(M, N) r(M, N) \subseteq P\}$. Thus $(M, N) P^{\prime \prime}(M, N) \subseteq P$. Since $P$ is a prime ideal of $R$ and does not contain $(M, N)$, it must be the case that $P^{\prime \prime} \subseteq P$. The reverse inclusion is obvious, and so $P=P^{\prime \prime}$.

THEOREM 2.3. Let $R$ be an essential subdirect sum of prime rings $\left\{R_{\alpha} \mid \alpha \in A\right\}$, and suppose that $(R, M, N, E)$ is a Morita context such that $(M e, N)=0$ only if $e=0 \in E .^{1}$ Then $E$ is an essential subdirect sum of prime rings $\left\{E_{\beta} \mid \beta \in B\right\}$ for some subset $B \subseteq A$.

Proof. $R$ is clearly semiprime and there exist prime ideals $\left\{P_{\alpha} \mid \alpha \in A\right\}$ of $R$ such that

(i) $R / P_{\alpha} \cong R_{\alpha}$ for each $\alpha \in A$,

(ii) $\bigcap_{\alpha} P_{\alpha}=0$,

(iii) $\bigcap_{\alpha \neq \beta} P_{\alpha} \neq 0$ for each $\beta \in A\left(R \cap R_{\beta} \neq 0\right)$.

Set $I=\{r \in R \mid r(M, N)=0\}$. $\quad I$ is an annihilator ideal, and we

${ }^{1}$ Equivalently, $E$ is semiprime and $[N, M]$ is an essential left ideal of $E$. 
assert that $I=\{r \in R \mid[N r, M]=0\}$. To see this, note that $r(M, N)=0$ implies that $(M[N r, M], N)=(M, N) r(M, N)=0$, whence by hypothesis $[N r, M]=0$; conversely, if $[N, r M]=0$ then $(r(M, N))^{2}=$ $(r M[N r, M], N)=0$ whence $r(M, N)=0$ because $R$ is semiprime.

Next, set $B=\left\{\beta \in A \mid P_{\beta} \supseteqq I\right.$ and $\left.P_{\beta} \nsupseteq(M, N)\right\}$ and $C=\left\{\gamma \in A \mid P_{\gamma} \supseteqq\right.$ $(M, N)\}$. Since each $P_{\alpha} \supseteqq I(M, N)=0$ and $P_{\alpha}$ is prime, it follows that $A$ is the disjoint union of $B$ and $C$. We will prove that $E$ is an essential subdirect sum of the prime rings $\left\{E_{\beta}=E / P_{\beta}^{\prime} \mid \beta \in B\right\}$; i.e., we show that (i) $\bigcap_{\beta \in B} P_{\beta}^{\prime}=0$, and (ii) $\bigcap_{\beta \neq \beta_{0}, \beta \in B} P_{\beta}^{\prime} \neq 0$ for any fixed $\beta_{0} \in B$.

(i) If $e \in \bigcap_{\beta \in B} P_{\beta}^{\prime}$, then $(M e, N) \subseteq \bigcap_{\beta \in B} P_{\beta}$, and so $(M e, N) \cong$ $\bigcap_{\beta \in B} P_{\beta} \cap \bigcap_{\gamma \in C} P_{\gamma}=\bigcap_{\alpha \in A} P_{\alpha}=0$. Thus $e=0$ and $\bigcap_{\beta \in B} P_{\beta}^{\prime}=0$.

(ii) Suppose $\bigcap_{\beta \neq \beta_{0}, \beta \in B} P_{\beta}^{\prime}=0$ for some $\beta_{0} \in B$. First observe that $I \cap \bigcap_{r \in C} P_{r} \leqq \bigcap_{\beta \in B} P_{\beta} \cap \bigcap_{r \in C} P_{r}=\bigcap_{\alpha \in A} P_{\alpha}=0$. But then

$$
\begin{aligned}
\bigcap_{\alpha \in A, \alpha \neq \beta_{0}} P_{\alpha} & =\bigcap_{\beta \in B, \beta \neq \beta_{0}} P_{\beta} \cap \bigcap_{\gamma \in C} P_{\gamma}=\bigcap_{\beta \in B, \beta \neq \beta_{0}} P_{\beta}^{\prime \prime} \cap \bigcap_{\gamma \in C} P_{\gamma} \\
& =\left\{r \in R \mid[N r, M] \subseteq \bigcap_{\beta \in B, \beta \neq \beta_{0}} P_{\beta}^{\prime}=0\right\} \cap \bigcap_{\gamma \in C} P_{\gamma}=I \cap \bigcap_{\gamma \in C} P_{\gamma}=0,
\end{aligned}
$$

where the second equality comes from Lemma 2.2. This contradicts $\bigcap_{\alpha \in A, \alpha \neq \beta_{0}} P_{\alpha} \neq 0$, and establishes the claim.

In case $C$ is the empty set (this occurs exactly when $I=0$ ), we define $\bigcap_{r \in C} P_{r}=0$, and the above proof is valid. Note that for the standard context with ${ }_{R} M$ torsionless we have $I=0$ exactly when ${ }_{R} M$ is faithful.

Corollary 2.4. Let $(R, M, N, E)$ be a Morita context such that $[N r, M]=0$ only if $r=0 \in R$. If $E$ is an essential subdirect sum of prime rings, then the same is true for $R$.

Proof. Apply the previous theorem to the symmetric Morita context $(E, N, M, R)$.

CoRollary 2.5. If ${ }_{R} M$ is a torsionless module and $R$ is an essential subdirect sum of prime rings, then the endomorphism ring of ${ }_{R} M$ is an essential subdirect sum of prime rings.

Proof. Use the standard context for ${ }_{R} M$.

CoRollary 2.6. If $R$ is a semiprime ring with the maximum condition on annihilator ideals, and $(R, M, N, E)$ is a Morita context such that $(M e, N)=0$ only if $e=0 \in E$, then $E$ is a semiprime ring with the maximum condition on annihilator ideals.

Proof. By [4, Theo. 3.13], $R$ is a semiprime ring with the 
maximum conditions on annihilator ideals if and only if $R$ is a subdirect sum of a finite number of prime rings. Theorem 2.3 ensures that this property is inherited by $E$.

REMARK. The methods employed above do not seem sufficient to treat the passage of chain conditions on one-sided annihilator ideals to endomorphism rings. Results in this direction would be of interest.

\section{REFERENCES}

1. H. Bass, The Morita Theorems, Lecture notes, University of Oregon, Eugene, Oregon, 1962.

2. J. J. Hutchinson, Intrinsic extensions of rings, Pacific J. Math., 30 (1969), 669-677.

3. - Quotient full linear rings, Proc. Amer. Math. Soc., 28 (1971), 375-378.

4. L. Levy, Unique subdirect sums of prime rings, Trans. Amer. Math. Soc., 106 (1963), 67-76.

Received March 13, 1972 and in revised form September 27, 1972. The second author was partially supported by NSF grant GP-34098.

WAShington STATE UNIVERSitY

AND

University of California, SANta Barbara 